\title{
Simulação do Escoamento de Películas Finas Aderidas a Superfícies Sólidas
}

\author{
Narcisa Corrêa da Silva ${ }^{1}$ \\ Luiz Nélio Henderson Guedes de Oliveira ${ }^{2}$ \\ Adriana da Rocha Silva ${ }^{3}$ \\ Janaína Imbiriba da Costa ${ }^{4}$ \\ Sérgio Silva de Sousa ${ }^{5}$ \\ Universidade do Estado do Rio de Janeiro, Instituto Politécnico - Nova Friburgo, RJ \\ Breno Tiago de Souza Mota ${ }^{6}$ \\ Universidade Federal do Oeste do Pará - UFOPA - Santarém, PA
}

\begin{abstract}
Resumo. O presente trabalho visa apresentar um esquema numérico para resolução de equações diferenciais parciais de alta ordem, as quais são não lineares. O problema aqui considerado surge em teoria da lubrificação, onde películas finas constituídas de fluidos viscosos deslocam-se em contato com superfícies sólidas. Tal problema é modelado por uma equação diferencial parcial parabólica não linear de quarta ordem. Este trabalho utiliza o chamado algoritmo espectral, o qual é um método para a solução de sistemas não lineares de grande porte, que não resolve sistemas lineares, nem usa qualquer informação explícita associada com a matriz Jacobiana. Os resultados obtidos mostram que este algoritmo moderno é um método eficiente para a simulação de escoamentos de películas finas aderidas a superfícies sólidas.
\end{abstract}

Palavras-chave. Método Espectral, Películas Finas, Equação Parabólica de Quarta Ordem, Sistemas Não lineares

\section{Introdução}

As películas líquidas finas aparecem em uma variedade de processos industriais. Podem ser compostas de líquidos comuns como água ou óleo, de materiais complexos tais como soluções de polímeros ou fusões, ou misturas complexas de fases ou componentes. Quando as películas são submetidas à ação de vários fatores mecânicos ou térmicos, exibem fenômenos dinâmicos interessantes, como propagação de ondas, intensificação de ondas e desenvolvimento de respostas caóticas [6]. Fluxos de película surgem na engenharia em processos de transferência de calor e massa e outras operações unitárias. Uma aplicação importante destes fluxos é o processo de revestimento. A tecnologia de revestimento é utilizada para fabricar diversos produtos, incluindo papéis, materiais de construção, tais como pavimentos e telhas, componentes eletrônicos, materiais de embalagem e filmes ópticos [4]. Estudos de métodos numéricos para a resolução de equações diferenciais parciais que modelam a evolução de películas finas é uma área da matemática aplicada, a qual se encontra

\footnotetext{
${ }^{1}$ narcisa.correa@gmail.com

${ }^{2}$ neliohenderson@gmail.com

3 adrianarochanf@gmail.com

4 janainaimbiriba@gmail.com

${ }^{5}$ pfsergiosousa@yahoo.com.br

${ }^{6}$ brenotsm1@gmail.com
} 
no início de seu desenvolvimento. Este trabalho tem como objetivo a modelagem e simulação computacional de um esquema numérico para a resolução de tais equações. A metodologia escolhida abordará métodos de diferenças finitas para problemas parabólicos não lineares, com condições de contorno de Neumann.

\section{Modelagem do Escoamento de Películas Finas}

Em muitas aplicações físicas, processos de evolução de películas finas de fluidos viscosos não são apenas influenciados pela tensão superficial existente entre o sólido e o líquido, mas também devido a diferentes efeitos físicos, como forças intermoleculares, por exemplo.

Em tais condições, o problema aqui considerado consiste em encontrar um par de funções $u, \mathcal{P}: \Omega \times(0, T) \rightarrow \mathbb{R}$, onde $\Omega$ é um conjunto limitado em $\mathbb{R}^{2}$, tal que

$$
\begin{gathered}
\frac{\partial u}{\partial t}-\nabla \cdot(\mathcal{M}(u) \nabla \mathcal{P})=0, \quad \text { em } \Omega \times(0, T), \\
\mathcal{P}=-\nabla^{2} u+w(u), \quad \text { em } \Omega \times(0, T), \\
\frac{\partial u}{\partial v}=\frac{\partial \mathcal{P}}{\partial v}=0, \quad \text { sobre } \partial \Omega \times(0, T), \\
u(x, y, t=0)=u_{0}(x, y), \quad \text { em } \Omega \times(0, T) .
\end{gathered}
$$

Aqui, $u$ representa a espessura da película de fluido, $\mathcal{P}$ é a pressão, $w(u)$ é um potencial externo usado para descrever diferentes efeitos físicos (não lineares) adicionais e $v$ denota o vetor unitário normal exterior à fronteira do domínio $\Omega$. A função $\mathcal{M}(u)$, chamada de mobilidade do fluido, é não negativa e anula-se em $u=0$. Este modelo matemático é derivado das Equações de Navier-Stokes para fluidos Newtonianos incompressíveis, veja por exemplo, as referências [6], [7] e [9].

Em geral, a mobilidade é uma função não linear, a qual depende das condições de contorno na superfície sólida. No presente trabalho, consideraremos a função de mobilidade do fluido dada por

$$
\mathcal{M}(u)=u^{3},
$$

o que é uma consequência da condição de não deslizamento na superfície sólida.

Em vista das Equações (1) e (2), notamos que as equações diferenciais deste modelo para o escoamento de películas finas podem também ser tratadas como um sistema de duas equações diferencias parciais com duas variáveis, dadas pelas funções $u$ e $\mathcal{P}$, que dependem da posição e do tempo. Neste trabalho, consideraremos o domínio $\Omega=\left[0, L_{x}\right] \times\left[0, L_{y}\right]$, onde $L_{x}$ e $L_{y}$ são as magnitudes dos lados desse domínio, medidas nas direções dos eixos coordenados $x$ e $y$, respectivamente.

A condição de contorno empregada na resolução das Equações (1) e (2) pode ser escrita da seguinte maneira

$$
\nabla u \cdot n=\nabla \mathcal{P} \cdot n=0, \text { sobre } \partial \Omega,
$$

em que $\partial \Omega$ é a fronteira de $\Omega$ e $n$ é o vetor unitário normal à $\partial \Omega$.

Considerando-se a ação das chamadas forças de Van der Waals (forças intermoleculares agindo no fluido), Witelski e Bowen [8] descrevem um simples modelo para as forças intermoleculares entre a superfície sólida e a película de fluido, a qual pode ser escrito pela função $w(u)$ e dada por

$$
w(u)=\left(\frac{1}{u^{3}}-\frac{\varepsilon}{u^{4}}\right) .
$$


Tendo em vista a Equação (4), usaremos as seguintes condições iniciais:

$$
\begin{aligned}
u_{0}(x, y) & =0,5+0,0025 \operatorname{sen}\left(6(x-0,65)^{2}\right)+0,0025 \operatorname{sen}\left(6(y-0,65)^{2}\right) \\
\mathcal{P}_{0}(x, y) & =0,03\left[12(x-0,65)^{2} \operatorname{sen}\left(6(x-0,65)^{2}\right)-\cos \left(6(x-0,65)^{2}\right)\right] \\
& +0,03\left[12(y-0,65)^{2} \operatorname{sen}\left(6(y-0,65)^{2}\right)-\cos \left(6(y-0,65)^{2}\right)\right] \\
& +\left(\frac{1}{\left(u_{0}(x, y)\right)^{3}}-\frac{\varepsilon}{\left(u_{0}(x, y)\right)^{4}}\right) .
\end{aligned}
$$

Tais condições descrevem uma película fina de altura 0,5 , ligeiramente perturbada por senoides, onde o parâmetro $\varepsilon$ estabelece uma escala para a espessura mínima da película.

\section{Metodologia}

O domínio espacial $\Omega=\left[0, L_{x}\right] \times\left[0, L_{y}\right]$ será discretizado utilizando uma grade de blocos centrados, denotada por $\hat{\Omega}=\left\{\left(x_{i}, y_{i}\right) \in \mathbb{R}^{2} ; i=1, \ldots, N_{x}\right.$ e $\left.j=1, \ldots, N_{y}\right\}$, onde os inteiros $N_{x}$ e $N_{y}$ indicam as quantidades de blocos na grade espacial, nas respectivas direções $x$ e $y$. Logo, fazendo-se $\Delta x=L_{x} / N_{x}$, pode-se escrever $x_{i}=(i-1 / 2) \Delta x$, para todo $i=1, \ldots, N_{x}$. De forma semelhante, tem-se $y_{j}=(j-1 / 2) \Delta y$, para todo $j=1, \ldots, N_{y}$, com $\Delta y=L_{y} / N_{y}$. O domínio temporal será representado pelo intervalo fechado $\left[t_{0}, t_{f}\right]$, onde $t_{0}$ denotará o instante inicial, considerado como sendo o tempo zero e $t_{f}$ o instante final.

Primeiramente consideraremos a Equação (1) discretizada implicitamente no tempo, com relação a $\mathcal{P}$ e $u$

$$
\frac{u_{i, j}^{n+1}-u_{i, j}^{n}}{\Delta t}-\left[\frac{\partial}{\partial x}\left(\mathcal{M}\left(u^{n+1}\right) \frac{\partial \mathcal{P}^{n+1}}{\partial x}\right)\right]_{i, j}-\left[\frac{\partial}{\partial y}\left(\mathcal{M}\left(u^{n+1}\right) \frac{\partial \mathcal{P}^{n+1}}{\partial y}\right)\right]_{i, j}=0 .
$$

Após a discretização por diferenças finitas centradas e algumas simplificações, obtém-se:

$$
\begin{array}{r}
\frac{u_{i, j}^{n+1}-u_{i, j}^{n}}{\Delta t}-\frac{1}{\Delta x} \times\left[\mathcal{M}_{i+\frac{1}{2}, j}\left(u^{n+1}\right) \frac{\mathcal{P}_{i+1, j}^{n+1}-\mathcal{P}_{i, j}^{n+1}}{\Delta x}-\mathcal{M}_{i-\frac{1}{2}, j}\left(u^{n+1}\right) \frac{\mathcal{P}_{i, j}^{n+1}-\mathcal{P}_{i-1, j}^{n+1}}{\Delta x}\right] \\
-\frac{1}{\Delta y} \times\left[\mathcal{M}_{i, j+\frac{1}{2}}\left(u^{n+1}\right) \frac{\mathcal{P}_{i, j+1}^{n+1}-\mathcal{P}_{i, j}^{n+1}}{\Delta y}-\mathcal{M}_{i, j-\frac{1}{2}}\left(u^{n+1}\right) \frac{\mathcal{P}_{i, j}^{n+1}-\mathcal{P}_{i, j-1}^{n+1}}{\Delta y}\right]=0 .
\end{array}
$$

Na Equação (11), o valor de $\mathcal{M}\left(u^{n+1}\right)$ em um ponto médio $i+\frac{1}{2}$, por exemplo, é calculado pela média harmônica dos valores $\mathcal{M}\left(u^{n+1}\right)$ nos pontos vizinhos $i+1$ e $i-1$. Agora, considerando-se a Equação (2) e o modelo que descreve as forças intermoleculares, temos que

$$
\mathcal{P}_{i, j}^{n+1}+\left[\frac{\partial}{\partial x}\left(\frac{\partial u^{n+1}}{\partial x}\right)\right]_{i, j}-\left[\frac{\partial}{\partial y}\left(\frac{\partial u^{n+1}}{\partial y}\right)\right]_{i, j}-w_{i, j}\left(u^{n+1}\right)=0,
$$

onde o termo $w_{i, j}\left(u^{n+1}\right)$ é dado pela seguinte equação

$$
w_{i, j}\left(u^{n+1}\right)=\left(\frac{1}{\left(u_{i, j}^{n+1}\right)^{3}}-\frac{\varepsilon}{\left(u_{i, j}^{n+1}\right)^{4}}\right) .
$$


No que segue, aproximando por diferenças centradas as derivadas parciais da Equação (12), obtém-se

$$
\begin{aligned}
\mathcal{P}_{i, j}^{n+1}+ & \frac{1}{\Delta x}\left[\frac{u_{i+1, j}^{n+1}-u_{i, j}^{n+1}}{\Delta x}-\frac{u_{i, j}^{n+1}-u_{i-1, j}^{n+1}}{\Delta x}\right] \\
+ & \frac{1}{\Delta y}\left[\frac{u_{i, j+1}^{n+1}-u_{i, j}^{n+1}}{\Delta y}-\frac{u_{i, j}^{n+1}-u_{i, j-1}^{n+1}}{\Delta y}\right]-w_{i, j}\left(u^{n+1}\right)=0 .
\end{aligned}
$$

As discretizações das equações diferenciais de interesse neste trabalho são transformadas em sistemas não lineares com $N=2 \times N_{x} \times N_{y}$ equações algébricas (ou transcendentes). Então, durante a evolução temporal, para obter-se os campos de espessura da película de fluido e a pressão, a resolução numérica de um sistema não linear é exigida a cada passo de tempo $\Delta t$ que separa o nível de tempo $n$ do nível futuro $n+1$. Os sistemas não lineares são resolvidos usando o método DFSANE (Derivative-Free Spectral Algorithm for Nonlinear Equations) proposto por La Cruz, Martínez e Raydan [5], o qual é um método espectral totalmente livre de derivadas, destinado à resolução de problemas não lineares de grande porte. Uma grande vantagem desta metodologia é que ela não requer o cálculo da matriz Jacobiana associada ao sistema não linear. De fato, este método espectral requer essencialmente a computação e armazenagem de dois vetores, que são, o vetor variável $x$ e o vetor de busca $F(x)$, ambos de tamanho $N=2 \times N_{x} \times N_{y}$. O método DFSANE foi implementado utilizando um código computacional feito em linguagem de programação $\mathrm{C}++$.

\section{Resultados}

Para estudar o caso da existência de forças intermoleculares entre a superfície sólida e a película de fluido, considera-se que o domínio espacial $\Omega$ é discretizado usando-se uma grade com $40 \times 40$ blocos, onde $\Delta x=\Delta y=0,025$ e o tamanho do passo de tempo usado foi de $\Delta t=10^{-5}$, o qual foi selecionado após intensivos testes computacionais. Além disso, em vista da Equação (7), consideraremos o parâmetro $\varepsilon=0,01$. Os quantificadores para a análise do desempenho do método DFSANE estão descritos na Tabela 1, para intervalos de tempo que variam de $T=10^{-5}$ até $T=0,10223$, os quais também foram escolhidos após intensivos testes computacionais.

Na Tabela 1, pode-se observar o número de passos de tempo NTS, o número total de avaliações de $F$ (a aplicação não linear associada com o sistema que representa a equação de película fina discretizada) no último passo de tempo, o número total de iterações realizadas pelo DFSANE na resolução do sistema não linear no último passo de tempo, o número total de etapas de globalização e o valor final da $\|F\|$ no último passo de tempo.

Tabela 1: Resultados para análise do desempenho do método DFSANE.

\begin{tabular}{ccccc}
\hline$T=\Delta t \times N T S$ & $\begin{array}{c}\text { Avaliações } \\
\text { de } F\end{array}$ & Iterações & $\begin{array}{c}\text { Etapas de } \\
\text { globalização }\end{array}$ & Norma de $F$ \\
\hline $10^{-5} \times 1=10^{-5}$ & 1506 & 748 & 758 & $2,092 \times 10^{-6}$ \\
$10^{-5} \times 10^{2}=10^{-3}$ & 91 & 71 & 20 & $3,264 \times 10^{-7}$ \\
$10^{-5} \times 10^{3}=10^{-2}$ & 65 & 47 & 18 & $8,292 \times 10^{-7}$ \\
$10^{-5} \times 10^{4}=10^{-1}$ & 1294 & 1020 & 274 & $2,144 \times 10^{-5}$ \\
$10^{-5} \times 10221=0,10221$ & 51969 & 35995 & 15974 & $1,831 \times 10^{-4}$ \\
$10^{-5} \times 10222=0,10222$ & 148063 & 112610 & 35432 & $2,093 \times 10^{-4}$ \\
$10^{-5} \times 10223=0,10223$ & 13384014 & $9 \times 10^{6}$ & 840200 & 26107,9 \\
\hline
\end{tabular}


Os resultados das simulações numéricas usando-se o DFSANE são ilustrados na Figura 1, para os instantes de tempo: $T=10^{-5}, T=10^{-1}$ e $T=0,10222$ e na Figura 2 para o instante de tempo $T=0,10223$.

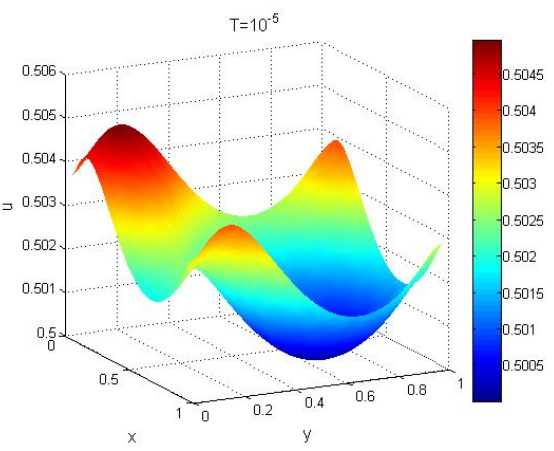

(a) $T=10^{-5}$

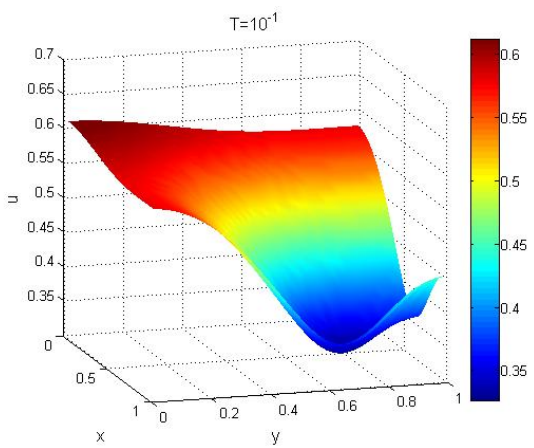

(c) $T=10^{-1}$

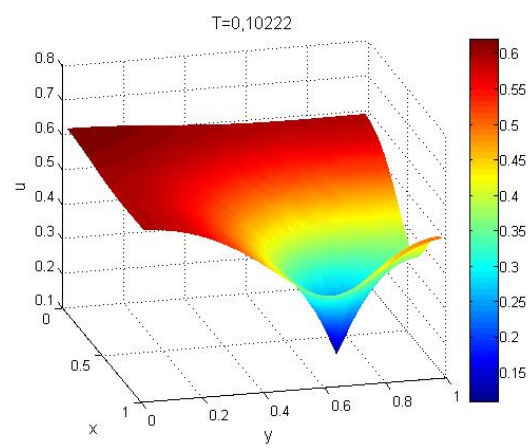

(e) $T=0,10222$



(b) $T=10^{-5}$

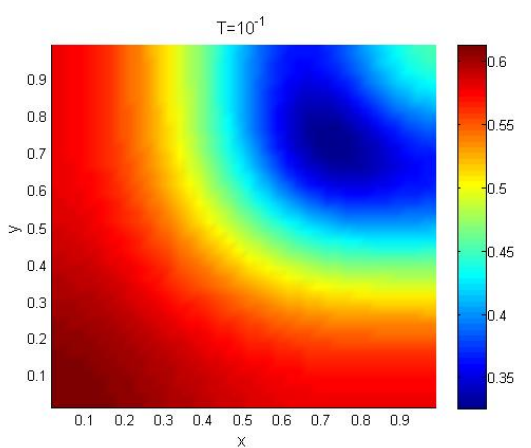

(d) $T=10^{-1}$

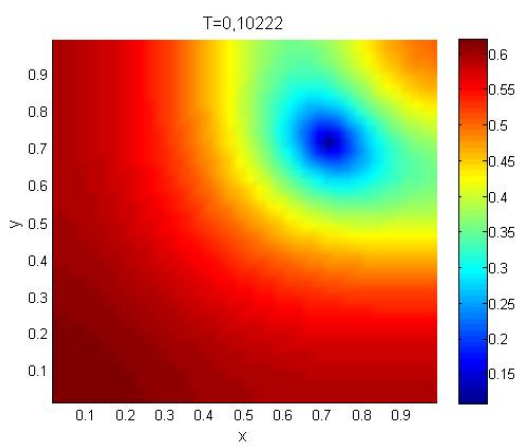

(f) $T=0,10222$

Figura 1: Superfícies da espessura da película de fluido e suas respectivas curvas de nível, em três diferentes instantes de tempo: $T=10^{-5}, T=10^{-1}$ e $T=0,10222$.

Observando-se a Figura 1, podemos notar que a película é muito suscetível à pequena perturbação inicial, diminuindo de valor em certas regiões do domínio e aumentando em outras. Esse comportamento é típico da ação de forças intermoleculares, e deve produzir rupturas na película, as quais se apresentam como singularidades associadas com a superfície solução do problema con- 
siderado. De fato, já na Figura 2 pode-se observar a superfície da espessura da película de fluido logo após a ruptura. Como enfatizado antes, tal fenômeno de ruptura ocorre devido à ação de forças intermoleculares, ou forças de Van der Waals, sendo também observado em experimentos realizados em laboratórios.
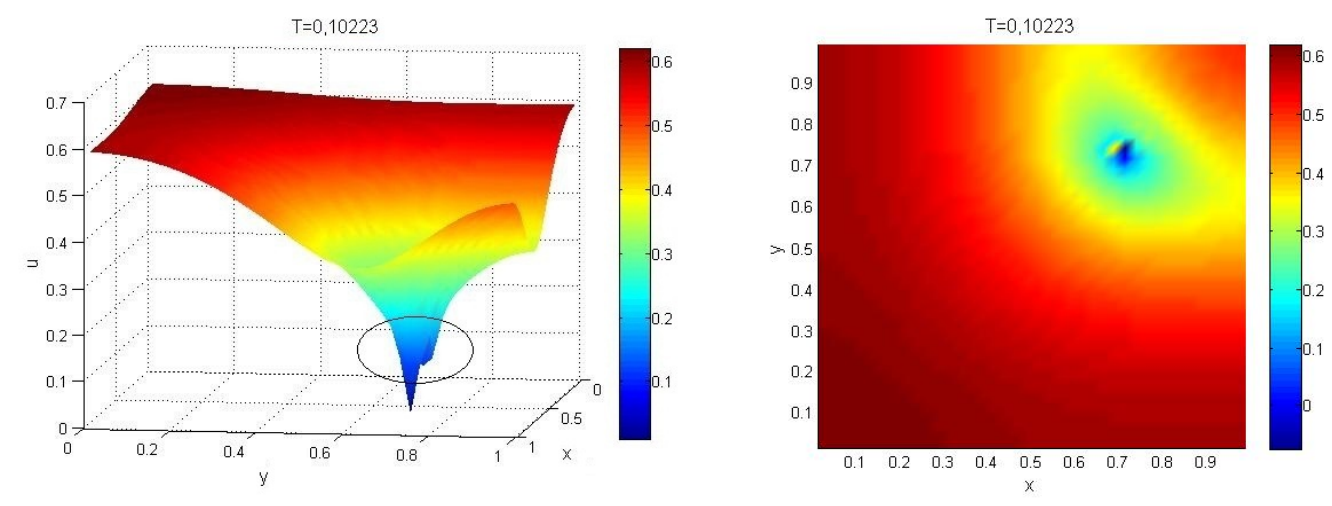

Figura 2: Superfície da espessura da película de fluido logo após a ruptura e sua respectiva curva de nível para o instante de tempo $T=0,10223$.

Como mostrado na Tabela 1 , podemos notar que no instante de tempo $T=0,10223$ a $\|F\|$ não atingiu um valor suficientemente pequeno, pois neste instante de tempo já havia ocorrido à ruptura da película de fluido, como pode ser observado na Figura 2, e o problema se tornou singular. Para capturar este fenômeno de ruptura, o DFSANE utilizou o número máximo de iterações considerado na simulação deste problema, isto é, $9 \times 10^{6}$ iterações para resolver o sistema não linear. Após as $9 \times 10^{6}$ iterações, a simulação numérica parou, não havendo então convergência, nem diminuição da $\|F\|$ no dado instante de tempo.

\section{Conclusões}

Neste trabalho resolveu-se numericamente um conjunto de equações diferenciais que modelam o problema do escoamento de películas finas aderidas em superfícies sólidas, o qual é de interesse da ciência aplicada e da engenharia. Avaliamos o comportamento das equações diferenciais parciais discretizadas por diferenças finitas, de forma implícita no tempo, obtendo um sistema não linear. Tal sistema não linear foi resolvido pela primeira vez empregando-se o chamado método DFSANE. Este método constitui uma abordagem livre de derivadas, o que diminui grandemente o esforço computacional, pois não calcula e também não armazena qualquer informação explícita associada com a matriz Jacobiana, e nem tão pouco resolve sistemas lineares.

A discretização das equações diferenciais parciais deram origem a um sistema não linear com 3200 variáveis que foi resolvido várias vezes, ao longo de muitos passos de tempo. Em relação ao desempenho do método numérico utilizado, os resultados obtidos permitem afirmar que o DFSANE foi capaz de simular o processo de evolução de películas finas de fluidos viscosos com a ação de forças intermoleculares, também conhecidas como forças de Van der Waals.

Assim pode-se concluir que o DFSANE é um método robusto e eficiente para a simulação do escoamento de películas finas em superfícies sólidas. 


\section{Agradecimentos}

O presente trabalho foi realizado com apoio da Coordenação de Aperfeiçoamento de Pessoal de Nível Superior - Brasil (CAPES) - Código de Financiamento 001.

Os autores também agradecem o apoio financeiro da Fundação de Amparo à Pesquisa do Estado do Rio de Janeiro - FAPERJ.

\section{Referências}

[1] Barzilai, J. and Borwein, J. M. Two-point step size gradient methods. IMA Journal of $\mathrm{Nu}$ merical Analysis, 8, 141-148, 1988. DOI: 10.1093/imanum/8.1.141.

[2] Becker, J., Grun, G. and Lenz, M., Bonn and Rumpf, M., Duisburg. Numerical methods for fourth order nonlinear degenerate diffusion problems. Applications of Mathematics, 47, 517-543, 2002. DOI: 10.1023/B:APOM.0000034537.55985.44.

[3] Bird, R. B., Stewart, W. E. and Lightfoot, E. N. Transport phenomena. Second edition. New York: Wiley, 2002.

[4] Kheshgi, H. S. Profile Equations for Film Flows at Moderate Reynolds Numbers. AIChE Journal, vol. 35, pp.1719-1727, 1989. DOI: 10.1002/aic.690351017.

[5] La Cruz, W., Martínez, J. M. and Raydan, M. Spectral residual method without gradient information for solving large-scale nonlinear systems of equations. Mathematics of Computation, vol. 75, 1429-1448, 2006. DOI: 10.1090/S0025-5718-06-01840-0.

[6] Oron, A., Davis, S. H. and Bankoff, S. G. Long-scale evolution of thin liquid films. Reviews of Modern Physics, vol. 69, 931-980, 1997. DOI: 10.1103/RevModPhys.69.931.

[7] Schlichting, H. and Gersten, K. Boundary-Layer Theory, 9 ed., Springer, 2016.

[8] Witelski, T. P. and Bowen, M. ADI schemes for higher-order nonlinear diffusion equations. Applied Numerical Mathematics, vol. 45, 331-351, 2003. DOI: 10.1016/S0168-9274(02)00194-0.

[9] Zhornitskaya, L. and Bertozzi, A. L. Positivity preserving numerical schemes for lubricationtype equations. SIAM J. Numer. Anal., 37, 523-555, 1999. DOI: 10.1137/S0036142998335698. 